\title{
Signal Diversification Is Associated with Corollary Discharge Evolution in Weakly Electric Fish
}

\author{
${ }^{\circledR}$ Matasaburo Fukutomi and ${ }^{\circledR}$ Bruce A. Carlson \\ Department of Biology, Washington University in St. Louis, St. Louis, Missouri 63130-4899
}

Communication signal diversification is a driving force in the evolution of sensory and motor systems. However, little is known about the evolution of sensorimotor integration. Mormyrid fishes generate stereotyped electric pulses (electric organ discharge [EOD]) for communication and active sensing. The EOD has diversified extensively, especially in duration, which varies across species from 0.1 to $>10 \mathrm{~ms}$. In the electrosensory hindbrain, a corollary discharge that signals the timing of EOD production provides brief, precisely timed inhibition that effectively blocks responses to self-generated EODs. However, corollary discharge inhibition has only been studied in a few species, all with short-duration EODs. Here, we asked how corollary discharge inhibition has coevolved with the diversification of EOD duration. We addressed this question by comparing 7 mormyrid species (both sexes) having varied EOD duration. For each individual fish, we measured EOD duration and then measured corollary discharge inhibition by recording evoked potentials from midbrain electrosensory nuclei. We found that delays in corollary discharge inhibition onset were strongly correlated with EOD duration as well as delay to the first peak of the EOD. In addition, we showed that electrosensory receptors respond to self-generated EODs with spikes occurring in a narrow time window immediately following the first peak of the EOD. Direct comparison of time courses between the EOD and corollary discharge inhibition revealed that the inhibition overlaps the first peak of the EOD. Our results suggest that internal delays have shifted the timing of corollary discharge inhibition to optimally block responses to self-generated signals.

Key words: animal communication; electrosensory system; evolution; sensorimotor integration; sensory coding

Significance Statement

Corollary discharges are internal copies of motor commands that are essential for brain function. For example, corollary discharge allows an animal to distinguish self-generated from external stimuli. Despite widespread diversity in behavior and its motor control, we know little about the evolution of corollary discharges. Mormyrid fishes generate stereotyped electric pulses used for communication and active sensing. In the electrosensory pathway that processes communication signals, a corollary discharge inhibits sensory responses to self-generated signals. We found that fish with long-duration pulses have delayed corollary discharge inhibition, and that this time-shifted corollary discharge optimally blocks electrosensory responses to the fish's own signal. Our study provides the first evidence for evolutionary change in sensorimotor integration related to diversification of communication signals.

\section{Introduction}

Diversification of communication signals is a driving force in animal speciation. Signal evolution has been associated with evolutionary changes to sensory receptors and central sensory circuits (Osorio and Vorobyev, 2008; Carlson et al., 2011; Baker et

\footnotetext{
Received Apr. 15, 2020; revised June 11, 2020; accepted July 6, 2020.

Author contributions: M.F. and B.A.C. designed research; M.F. performed research; M.F. analyzed data; M.F. and B.A.C. wrote the paper.

This work was supported by National Science Foundation Grant IOS-1755071 to B.A.C., and the Uehara Memorial Foundation to M.F. We thank Carl D. Hopkins and Natalie Trzcinski for kindly providing their Knollenorgan recording data; Adalee Lube for assistance with electrophysiology; and Erika Schumacher for assistance with statistical analysis.

The authors declare no competing financial interests.

Correspondence should be addressed to Bruce A. Carlson at carlson.bruce@wustl.edu.

https://doi.org/10.1523/JNEUROSCI.0875-20.2020

Copyright $\odot 2020$ the authors
}

al., 2015; ter Hofstede et al., 2015; Vélez and Carlson, 2016; Silva and Antunes, 2017; Vélez et al., 2017; Seeholzer et al., 2018), as well as peripheral effectors and central motor circuits (Bass, 1986; Otte, 1992; Podos, 2001; Paul et al., 2015; Ding et al., 2019; Jacob and Hedwig, 2019; Kwong-Brown et al., 2019). Despite this accumulated knowledge of sensory and motor system evolution, we know little about the evolution of sensorimotor interactions between these systems.

Corollary discharges are one of the links by which motor control influences sensory processing to distinguish external from self-generated stimuli (Sperry, 1950; von Holst and Mittelstaedt, 1950; Poulet and Hedwig, 2007; Crapse and Sommer, 2008; Schneider and Mooney, 2018; Straka et al., 2018). For communicating animals, a corollary discharge generally works to filter out an animal's own signals (reafference), allowing selective sensory processing of signals from other individuals (exafference). Since 
a corollary discharge needs to selectively cancel reafferent input, its function should evolve to adapt to signal diversification. However, this question has not been addressed to our knowledge.

Here we investigate corollary discharge evolution in mormyrids, African weakly electric fishes. These fish produce electricpulse signals, termed electric organ discharge (EOD), which are used for electrolocation and communication (Hopkins, 1999; von der Emde, 1999). EOD waveforms are stereotyped but diverse among species and sometimes among individuals within species (Hopkins, 1981; Arnegard et al., 2010; Paul et al., 2015; Gallant and O'Connell, 2020). The EOD is generated from an electric organ (EO) at the base of the tail (Fig. 1A) (Bennett, 1971). EOD waveform is determined by the biophysical characteristics of electrocytes in the EO, while EOD timing is determined by central neural commands (Bennett, 1971; Bass, 1986; Carlson, 2002a).

Electric communication signals are processed by a dedicated sensory pathway (Fig. 1A) (Xu-Friedman and Hopkins, 1999; Baker et al., 2013). Sensory receptors called knollenorgans (KOs) respond to outside-positive changes in voltage across the skin, or inward current, with a fixed latency spike (Bell, 1989). Because each $\mathrm{KO}$ faces out toward the surrounding water, in response to an external EOD, KOs on one side of the body receive an inward current, whereas KOs on the other side receive an outward current (Hopkins and Bass, 1981). By contrast, in response to a selfgenerated EOD, KOs on both sides receive the same-direction currents of the waveform consisting of a large outward current followed by a large inward current if the EOD has a head-positive polarity (Fig. 1A). The $\mathrm{KO}$ afferent fibers project to the nucleus of the electrosensory lateral line lobe (nELL) in the hindbrain, where corollary discharge inhibition (CDI) blocks responses to the fish's own EOD (Fig. 1A) (Bell and Grant, 1989). The axons of nELL neurons project to the anterior exterolateral nucleus (ELa) of the midbrain torus semicircularis, which sends its only output to the posterior exterolateral nucleus (ELp) (Fig. 1A) (Xu-Friedman and Hopkins, 1999).

Mormyrids are advantageous for studying corollary discharge interactions between motor and sensory systems: the motor command signal (fictive EOD) can be easily recorded from spinal electromotor neurons (EMNs) when EOD production is silenced pharmacologically. Previous studies reported that CDI starts $\sim 2 \mathrm{~ms}$ after the onset of a command signal and lasts for $\sim 2$ ms (Fig. 1B) (Amagai, 1998; Lyons-Warren et al., 2013b; Vélez and Carlson, 2016). Those studies used limited species that have short-duration EODs ( $\sim 0.5 \mathrm{~ms})$, but the mormyrid family has evolved EOD durations ranging from 0.1 to $>10 \mathrm{~ms}$ (Hopkins, 1999). The present study uses 7 mormyrid species having EODs that vary in duration from $\sim 0.1$ to $\sim 10 \mathrm{~ms}$, and compares CDI across species to reveal evolutionary change of corollary discharge in relation to signal diversification.

\section{Materials and Methods}

All procedures were in accordance with guidelines established by the National Institutes of Health and were approved by the Animal Care and Use Committee at Washington University in St. Louis.

Animals. Six Brienomyrus brachyistius (standard length [SL] $=7.5-$ $11.8 \mathrm{~cm}), 3$ Brevimyrus niger $(\mathrm{SL}=6.7-9.5 \mathrm{~cm}), 3$ Campylomormyrus compressirostris $(\mathrm{SL}=11.8-14.0 \mathrm{~cm}), 6$ Campylomormyrus numenius $(\mathrm{SL}=12.4-14.2 \mathrm{~cm}), 4$ Campylomormyrus tamandua $(\mathrm{SL}=6.5-9.1 \mathrm{~cm})$, 3 Gnathonemus petersii $(\mathrm{SL}=10.2-11.6 \mathrm{~cm})$, and 2 Mormyrus tapirus $(\mathrm{SL}=12.3-12.3 \mathrm{~cm})$ contributed EOD and evoked potential data to this study. We used fish of both sexes in B. brachyistuis, B. niger, C.
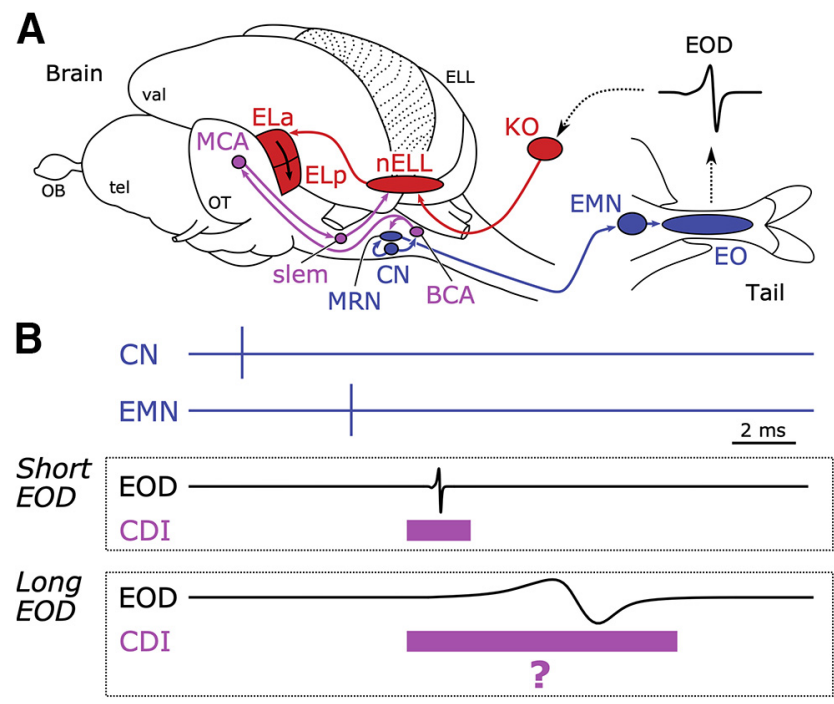

Figure 1. Corollary discharge inhibition (CDI) and evolution of EOD duration. $\boldsymbol{A}$, Diagram showing electromotor (blue), knollenorgan (KO) sensory (red), and corollary discharge (purple) pathways. The command nucleus (CN) drives the electric organ (E0) to generate each EOD via the medullary relay nucleus (MRN) and spinal electromotor neurons (EMN). K0 electroreceptors receive the EOD and send time-locked spikes to the nucleus of the electrosensory lateral line lobe (nELL) via primary afferents. The nELL neurons project their axons to the anterior exterolateral nucleus (ELa), which sends its only output to the adjacent posterior exterolateral nucleus (ELp). The CN provides another output to the bulbar command-associated nucleus $(B C A)$, which in turn projects to the MRN and to the mesencephalic command-associated nucleus (MCA). The MCA sends its output to the sublemniscal nucleus (slem) that has GABAergic neurons projecting to the nELL. ELL, electrosensory lateral line lobe; $\mathrm{OB}$, Olfactory bulb; $\mathrm{OT}$, optic tectum; tel, telencephalon; val, valvula of the cerebellum. $\boldsymbol{B}$, Potential hypothesis of $\mathrm{CDI}$ between mormyrids with short EODs and long EODs. The schematic diagram shows spike timings of the $\mathrm{CN}$ and EMN as well as EODs. The purple rectangles represent the potential time windows of $C D I$. In the short-EOD species, the corollary discharge covers the KO spike to selfgenerated EODs so as to inhibit sensory responses in the nELL. Since long-duration EODs can cause KO spikes with different timing, CDI needs to change its duration and/or timing to block reafferent responses in the nELL.

numenius, and C. tamandua, but only female in C. compressirostris, $G$. petersii, and M. tapirus. Subsets of these fish (1 B. brachyistius, 1 C. compressirostris, and 1 C. numenius) were used for simultaneous recording of the EOD and EOD command generated by EMNs. All fish were purchased from Bailey Wholesale Tropical Fish or AliKhan Tropical Fish. The fish were housed in water with a conductivity of $175-225 \mu \mathrm{S} / \mathrm{cm}$, a $\mathrm{pH}$ of $6-7$, and a temperature of $25-29^{\circ} \mathrm{C}$. The fish were kept on a $12 / 12$ $\mathrm{h}$ light/dark cycle and fed live black worms 4 times a week.

EOD recording and analysis. We recorded 10 EODs from each fish while it was freely swimming. EODs were amplified 10 times, bandpass filtered ( $1 \mathrm{~Hz}$ to $50 \mathrm{kHz})$ (BMA-200, Ardmore), digitized at a rate of $195 \mathrm{kHz}$ (RP2.1, Tucker Davis Technologies), and saved using custom software in MATLAB (The MathWorks).

EODs generally consist of peak 1 (maximum head-positive peak) and peak 2 (maximum head-negative peak). Some species we recorded from (B. brachyistius, B. niger, C. tamandua, and G. petersii) have EODs with an additional peak 0 (small head negative peak before peak 1). For EODs without a peak 0, EOD onset was determined as the point crossing $2 \%$ of peak- 1 amplitude. For EODs with a peak 0 , EOD onset was determined as the point crossing $20 \%$ of peak- 0 amplitude. In both cases, EOD offset was determined as the point crossing $2 \%$ of peak- 2 amplitude. EOD duration was determined as the period between EOD onset and offset. Delay to peak 1 was determined as the period between EOD onset and timing of peak 1. In addition, EOD frequency content was calculated by fast Fourier transformation.

We grouped EODs of C. numenius into three arbitrary types based on variation in duration (long, intermediate, and short EOD), following a previous study that revealed extensive individual variation in EOD duration within this species (Paul et al., 2015). In the following analysis, we used the average value without separating types. 
Simultaneous recording and analysis of EOD and EOD command. This recording session was performed after EOD recording and before evoked potential recording only for 3 fish. Fish were anesthetized in a solution of $300 \mathrm{mg} / \mathrm{L}$ tricaine methanesulfonate (MS-222) (Sigma Millipore) and positioned on a plastic platform in a recording chamber filled with fresh water. Fish were restrained by lateral plastic pins and a plastic tube on the tail. Freshwater was provided through a pipette tip in the fish's mouth. EOD commands from spinal EMNs were recorded with a pair of electrodes located within the plastic tube and oriented parallel to the fish's EO, amplified 1000 times, and bandpass filtered $(10 \mathrm{~Hz}$ to $5 \mathrm{kHz}$ ) (model 1700, A-M Systems). While EOD commands from EMNs were recorded, the EODs were recorded by separate electrodes, amplified 10 times, and bandpass filtered $(1 \mathrm{~Hz}$ to $50 \mathrm{kHz})$ (BMA-200, Ardmore). These recordings were digitized at a rate of $1 \mathrm{MHz}$ and saved (TDS 3014C, Tektronix). We recorded 9-11 trials from each fish.

EOD command traces from EMNs were averaged across trials, and EOD traces were filtered by a 21st-order median filter whose time window was $0.02 \mathrm{~ms}$ and averaged across trials. EOD onset was determined in the same way we determined EOD onset in freely swimming EOD recordings. EOD command onset was determined as the first negative peak in the averaged EMN trace. Delay to EOD onset ( $\left.\mathrm{D}_{\text {Onset }}\right)$ was calculated as the period between EOD command onset and EOD onset. Delay to peak 1 of EOD $\left(D_{\mathrm{P} 1}\right)$ from EOD command was calculated as the sum of $\mathrm{D}_{\text {Onset }}$ and the delay between EOD onset and peak 1 recorded from freely swimming fish.

Surgery and evoked potential recording. This recording session was performed after EOD recording (or simultaneous recording of EODs and EOD commands for the 3 fish). We prepared fish for in vivo recordings from ELa and ELp as described previously (Carlson, 2009; LyonsWarren et al., 2013a). Briefly, fish were anesthetized in $300 \mathrm{mg} / \mathrm{L}$ MS-222 and paralyzed with an intramuscular injection of $0.1 \mathrm{ml}$ of $3.0 \mathrm{mg} / \mathrm{ml}$ gallamine triethiodide (Flaxedil, Sigma Millipore). The fish were then transferred to a recording chamber $(20 \times 12.5 \times 45 \mathrm{~cm})$ filled with water and positioned on a plastic platform, leaving a small region of the head above water level. During surgery, we maintained general anesthesia by respirating the fish with an aerated solution of $100 \mathrm{mg} / \mathrm{ml} \mathrm{MS}$ 222 through a pipette tip placed in the mouth. For Campylomormyrus species, we connected a hose made from heat shrink tubing to the pipette tip so as to provide respiration to the long, tube-like mouth. For local anesthesia, we applied $0.4 \%$ lidocaine on the skin overlying the incision site, and then made an incision to uncover the skull overlying the ELa and ELp. Next, we glued a headpost to the skull before using a dental drill and forceps to remove a rectangular piece of skull covering the ELa and ELp. In Campylomormyrus species, the ELa and ELp are not exposed superficially, so we exposed ELa and ELp by separating the optic tectum and the valvula cerebellum using two retractors made from borosilicate capillary glass (Vélez and Carlson, 2016; Vélez et al., 2017). After exposing ELa and ELp, we placed a reference electrode on the nearby cerebellum. Following surgery, we switched respiration to fresh water and allowed the fish to recover from general anesthesia. We monitored the anesthetized state of the fish with a pair of electrodes oriented parallel to its $\mathrm{EO}$ within a plastic tube to record fictive EOD commands produced by the EMNs (Carlson, 2009; Lyons-Warren et al., 2013a). These EOD commands were $1000 \times$ amplified (model 1700, A-M Systems) and sent to a window discriminator for time stamping (SYS-121, World Precision Instruments). At the end of the recording session, the respiration of the fish was switched back to $100 \mathrm{mg} / \mathrm{L}$ MS-222 until no fictive EOD could be recorded, and then the fish was killed by freezing.

Evoked potentials in ELa and ELp were obtained with glass microelectrodes made of borosilicate capillary glass (o.d. $=1.0 \mathrm{~mm}$, i.d. $=0.5$ $\mathrm{mm}$; model 626000, A-M Systems) pulled on a micropipette puller (model P-97, Sutter Instrument), broken to a tip diameter of 10-20 $\mu \mathrm{m}$, and filled with $3 \mathrm{~m} \mathrm{NaCl}$ solution. Evoked potentials were $1000 \times$ amplified, bandpass filtered $(10 \mathrm{~Hz}-5 \mathrm{kHz})$ (model 1700, A-M Systems), digitized at a rate of $97.7 \mathrm{kHz}$ ( $\mathrm{RX} 8$, Tucker Davis Technologies), and saved using custom software in MATLAB (The MathWorks).

Sensory stimulation. We used three vertical electrodes on each side of the recording chamber (anodal to the fish's left, cathodal to the right) to deliver transverse stimuli with normal polarity (peak preceding trough). Digital stimuli were generated using custom software in MATLAB, converted to analog signals with a signal processor (RX8, Tucker Davis Technologies), attenuated with an attenuator (PA5, Tucker Davis Technologies), and isolated from ground with a stimulus isolation unit (model 2200, A-M Systems).

To examine CDI of sensory responses, we delivered $0.2 \mathrm{~ms}$ bipolar square pulses at several delays following the EOD command onset. Because KOs respond with time-locked spikes to the edges of square pulses (Bennett, 1965; Hopkins and Bass, 1981; Lyons-Warren et al., 2012; Baker et al., 2015), this short square-pulse stimulation allowed us to precisely control the timing of sensory input to the $\mathrm{KO}$ system and quantify the timing of CDI in nELL. The EOD command onset was determined as the first negative peak of the EOD command waveform that consists of a three-spike potential resulting from the synchronous activation of EMNs. Each delay was repeated 10 times, and the averaged response was used for analysis. First, we recorded sensory responses in the ELp at delays between 0 and $20 \mathrm{~ms}$ in $0.5 \mathrm{~ms}$ steps. Based on these initial data, we used custom software written in $\mathrm{R}$ to determine the range of delays to examine CDI with higher time resolution. The algorithm included the following steps: (1) calculated peak-to-peak amplitudes as a measure of response across all delays, (2) normalized all responses to the maximum response, (3) determined the latency resulting in the minimum response amplitude, (4) determined the onset and offset delays that resulted in responses just $<80 \%$ of the maximum response, and (5) determined the range of delays to examine CDI with higher time resolution as $2 \mathrm{~ms}$ before the onset time to $2 \mathrm{~ms}$ after the offset time. Then, we recorded sensory responses in the ELp to stimulus delays across this range in 51 equally spaced steps $(\sim 0.1-0.2 \mathrm{~ms})$. After recording in the ELp, we recorded sensory responses in the ELa using the same stimulus delays used in both the broad and narrow ranges tested in ELp. The stimulus sequences were randomized in all recordings.

To observe clear CDI, we needed to decide on an adequate stimulus intensity for each tested individual. First, we recorded evoked potentials at $0,3,4$, and $5 \mathrm{~ms}$ delays at $20 \mathrm{~dB}$ attenuation (reference intensity of $736 \mathrm{mV} / \mathrm{cm}$ ) and determined peak-to-peak amplitudes at each delay. Then, we calculated ratios of peak-to-peak amplitudes at 3, 4, and $5 \mathrm{~ms}$ delay to the one at $0 \mathrm{~ms}$ delay. If the minimum ratio was $<30 \%$, we chose this stimulus intensity. If the ratio was $>30 \%$, we reduced the intensity by adding $5 \mathrm{~dB}$ attenuation and performed the above procedure until the ratio got to $<30 \%$. From this procedure, we chose $23.4 \mathrm{mV} / \mathrm{cm}$ for 1 C. compressirostris and $73.6 \mathrm{mV} / \mathrm{cm}$ for all the other fishes.

Evoked potential recording analysis. We characterized CDI with respect to timing and duration. All analyses here were done using the recordings from the high-resolution, narrow range of stimulus delays. Normalized amplitude was calculated by the following steps: (1) calculated peak-to-peak amplitude for each delay, (2) subtracted the minimum peak-to-peak amplitude across all delays, and (3) divided by the maximum peak-to-peak amplitude across all delays, which leads to setting the maximum value as 1 and the minimum value as 0 . Then, we set an $80 \%$ threshold to determine the inhibition onset, offset, and duration. In addition, we determined the peak time of inhibition as the stimulus delay at which the response amplitude was minimal.

$K O$ recording and analysis. $\mathrm{KO}$ recording data from $B$. brachyistius $(n=6 \mathrm{KOs}), B$. niger $(n=5)$, Campylomormyrus alces $(n=1)$, C. compressirostris $(n=7), C$. numenius $(n=2)$, and C. tamandua $(n=2)$ came from previously published studies (Trzcinski, 2008; Trzcinski and Hopkins, 2008; Lyons-Warren et al., 2012; Baker et al., 2015). Based on a recent study of Campylomormyrus species (Paul et al., 2015), we concluded that Campylomormyrus sp. B shown in Trzcinski (2008) and Trzcinski and Hopkins (2008) was C. numenius.

The recording methods were generally shared among these previous studies. Similar to our evoked potential recording, fish were immobilized with Flaxedil, transferred to a recording chamber filled with freshwater, and positioned on a plastic platform with lateral support. The fish were provided freshwater through a pipette tip in the fish's mouth while monitoring the fish's EOD command signals using a pair of electrodes placed next to the fish's tail. Extracellular recordings of $\mathrm{KO}$ spikes were made using a wire electrode inside glass capillary tubing that was placed directly next to a KO. The signals were amplified, digitized, and recorded with 
custom software in MATLAB. Sensory stimuli consisted of conspecific EOD waveforms generated in MATLAB, digital-to-analog converted, attenuated, and delivered as a constant-current stimulus through the electrode.

These previous studies recorded $\mathrm{KO}$ responses to multiple waveforms at several intensities. For each recording, relative but not absolute intensities were known because the amount of current going into the electroreceptor pore is dependent on the position of the electrode tip relative to the pore, the size and shape of the pore, and the resistive paths between the electrode tip and the pore. Importantly, however, self-generated EODs will be of relatively large intensity. We therefore chose among these data here using the following criteria: (1) the EOD waveform stimulus was the inverted form of a conspecific EOD recorded with recording electrode at the head and reference electrode at the tail to simulate the self-generated EOD waveform; and (2) the largest stimulus intensity tested that did not result in a stimulus artifact that exceeded $\mathrm{KO}$ spike amplitude.

To calculate normalized $\mathrm{KO}$ responses, we made peristimulus time histograms (bin size $=0.02048 \mathrm{~ms}$ ) of KO spikes and normalized them by the maximum spike counts. Delay to peak KO response was determined as the period between the onset of the EOD stimulus and the time of the maximum $\mathrm{KO}$ response.

Experimental design and statistical analysis. The primary objective of this study was to determine the relationship between EOD waveform and CDI. We used 7 species, including 27 fishes, and recorded EODs from freely swimming individuals followed by evoked potential recording from the midbrain. Subsequently, we asked whether CDI overlapped KO spike timing to block responses to self-generated EODs. To compare the time courses between CDI and EOD using an identical reference of EOD command onset, we performed simultaneous recording of EOD and EOD command from a subset ( 3 species including 3 fishes) of them before performing electrophysiology. Furthermore, we measured KO response latency to self-generated EODs using previously published data (Trzcinski, 2008; Trzcinski and Hopkins, 2008; Lyons-Warren et al., 2012; Baker et al., 2015).

Although CDI occurs in nELL (Mugnaini and Maler, 1987; Bell and Grant, 1989), we focused on the downstream pathway, ELa and ELp. There are two reasons for this. First, neural recordings from nELL are much more challenging because it is a deep structure, whereas ELa and ELp are superficial structures. Second, recordings from nELL reveal complex evoked potentials consisting of sensory responses time-locked to the stimulus as well as CDI time-locked to the EOD command, and these occur at different relative times for different stimulus delays (Bell and Grant, 1989). By contrast, recordings from the downstream target of nELL allow us to isolate sensory evoked potentials from corollary discharge potentials and measure the effects of CDI in nELL (Russell and Bell, 1978). We recorded from both ELa and ELp because the present study includes several species (C. compressirostris, C. numenius, C. tamandua, and M. tapirus) for which evoked potential recordings from ELa and ELp had never before been published. We therefore recorded from both nuclei in all individuals studied to compare among the species and determine whether the response characteristics of ELa and ELp in unstudied species were similar to previously studied species.

Here we recorded evoked field potentials rather than single-unit spiking activities. Individual neurons in ELa and ELp show diversity both in terms of cellular types (afferents, efferents and interneurons) and sensory tuning within the types (Carlson, 2009; Baker et al., 2013; Lyons-Warren et al., 2013b). This would require very large numbers of recordings to accurately capture the corollary discharge effects. Field potential recording is a reliable and relatively simple technique that provides valuable insights into integrative processes within brain nuclei (Einevoll et al., 2013). Although the biophysical basis of local field potentials is disputed, it clearly represents summated electrical activity of neurons, which may reflect neuronal spiking and synaptic activity in the vicinity of the recording electrode. The aim of the present study was not to quantify the strength of inhibition in individual neurons, but the timing and duration of inhibitory effects across the population. Indeed, many previous studies used evoked potential recordings from ELa and ELp and showed clear and reliable CDI in some mormyrid species (Russell and Bell, 1978; Amagai, 1998; Lyons-Warren et al., 2013b; Vélez and Carlson, 2016). Therefore, evoked field potential recording is ideal for the purposes of the present study.
For statistical analysis, we used a phylogenetic generalized least squares (PGLS) model with a Brownian correlation structure to account for phylogenetic effects on the correlation analyses. For cross-species correlation analyses, it is necessary to incorporate phylogenetic information into a model because the lack of independence between data points of varying relatedness violates the assumptions of standard linear regression (Grafen, 1989; Mundry, 2014). We used a previously constructed bootstrapped maximum-likelihood tree from 73 Cytb osteoglossomorph sequences (Sullivan et al., 2000; Lavoué et al., 2003; Feulner et al., 2008; Sukhum et al., 2018). To include data from species that have not been sequenced, we used sequence data from within monophyletic genera and chose the species sequence with the shortest phylogenetic distance from the genus node. In this analysis, we used average values within species of EOD waveform, CDI, and KO response. Using this PGLS model, we estimated both the slope and the intercept of the regression line and calculated $t$ values, $p$ values, and 95\% CIs for each parameter. We used the $t$ and $p$ values to determine whether a given parameter was significantly different from zero. All phylogenetic analyses were performed in R programming software with the ape and nlme packages (Paradis and Schliep, 2019; Pinheiro et al., 2020). To examine individual differences within C. numenius, we used standard linear regression analysis rather than PGLS.

\section{Results}

\section{Mormyrids have diverse species-specific EODs}

To relate CDI to EOD waveform, we recorded EODs individually from 7 species before performing evoked potential recordings (Fig. 2A). EOD duration varied widely across species, from as short as $0.17 \mathrm{~ms}$ in C. compressirostris to as long as $8.59 \mathrm{~ms}$ in $C$. numenius (Fig. 2B). Fast Fourier transformation revealed that peak power frequencies ranged from $110 \mathrm{~Hz}$ in $C$. numenius to $7610 \mathrm{~Hz}$ in C. compressirostris (Fig. 2C,D).

\section{Corollary-discharge timing and duration vary among species} To measure the inhibitory effect of corollary discharge in the KO pathway, we performed evoked potential recordings from ELa and ELp (Fig. $3 A$ ). We stimulated with $0.2 \mathrm{~ms}$ bipolar square electric pulses delivered with a delay of $0-20 \mathrm{~ms}$ following the EOD command from spinal EMNs (Fig. 3A). To our knowledge, these are the first evoked potential recordings from the midbrain of Campylomormyrus species and M. tapirus. The recording traces of evoked potentials from those species were similar to those of previously reported species, including B. brachyistius, B. niger, G. petersii, Petrocephalus microphthalmus, and Petrocephalus tenuicauda (Russell and Bell, 1978; Amagai, 1998; Carlson, 2009; Lyons-Warren et al., 2013b; Vélez and Carlson, 2016): electrosensory stimulation elicited sharp and short-latency ( $\sim 2-4 \mathrm{~ms})$ evoked potentials in ELa, and broad and longer-latency $(\sim 6-$ $10 \mathrm{~ms}$ ) evoked potentials in ELp (Fig. 3B). We also tested whether response latencies in ELa to the short square-pulse stimulus was correlated with EOD duration among species, but the response latency was independent of EOD duration (estimated slope $=0.01 \mathrm{~ms} / \mathrm{ms}, 95 \% \mathrm{CI}=-0.08$ to $0.10 \mathrm{~ms} / \mathrm{ms}, t_{(5)}=$ $0.37, p=0.73$; estimated intercept $=2.9 \mathrm{~ms}, 95 \% \mathrm{CI}=2.3-$ $\left.3.6 \mathrm{~ms}, t_{(5)}=11.7, p=0.0001\right)$.

In each species, we found a narrow range of stimulus delays for which electrosensory responses were blocked by CDI (Fig. $3 C$ ), as shown previously in B. brachyistius and B. niger (Amagai, 1998; Lyons-Warren et al., 2013b; Vélez and Carlson, 2016). From these evoked potential traces, we determined the CDI window for each individual using normalized amplitudes and an $80 \%$ cutoff line (Fig. $3 D$ ). This revealed that corollary discharge onset, offset, duration, and peak time varied among species (Fig. $3 D$ ). For example, evoked potentials in the short-EOD C. compressirostris were blocked when sensory stimuli were delivered with a 3-4 ms delay following the EOD command, whereas 
A

$$
\text { C. numenius (Long EOD) }
$$

(Intermediate EOD)

(Short EOD)

M. tapirus

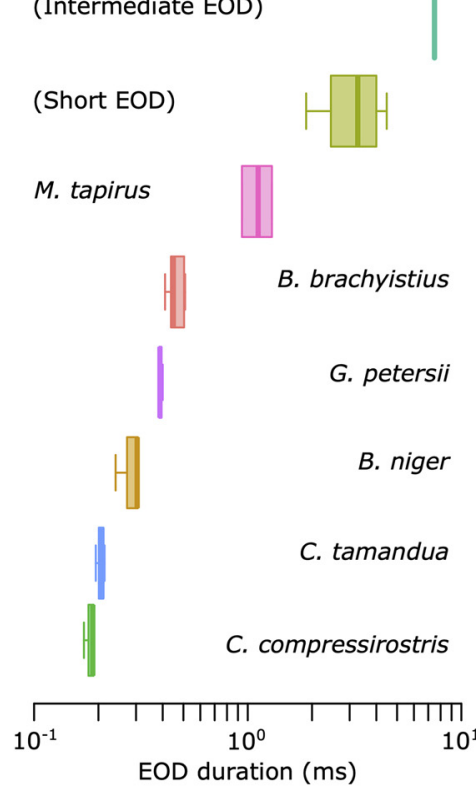

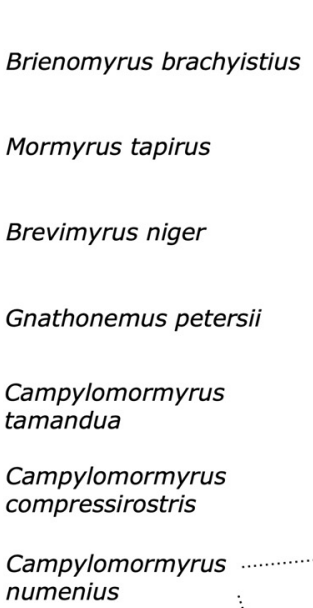
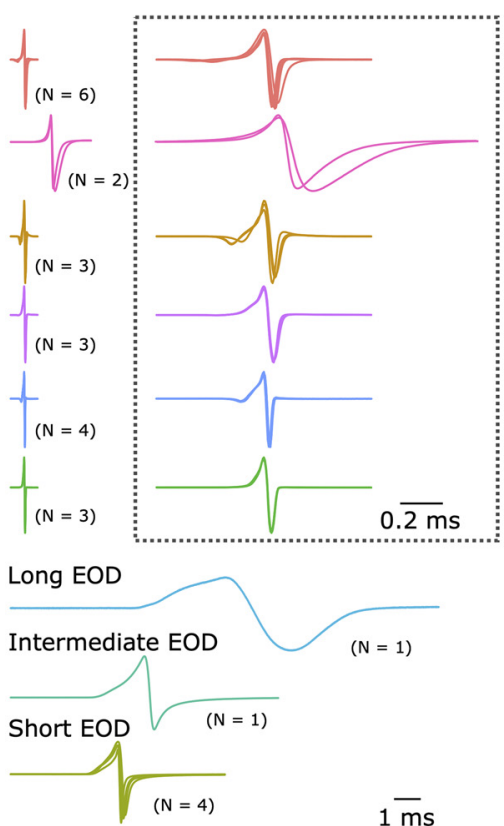

C

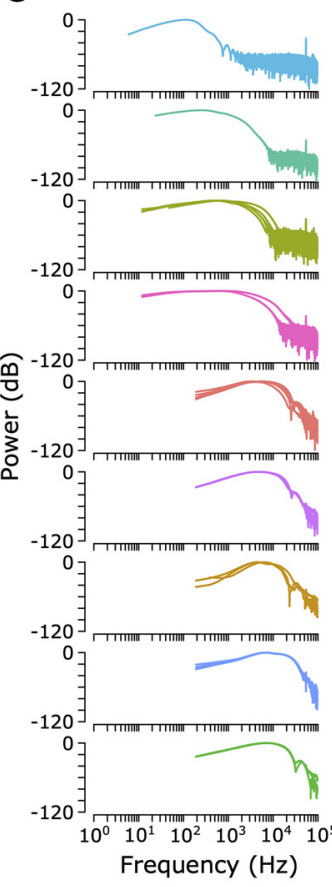

D

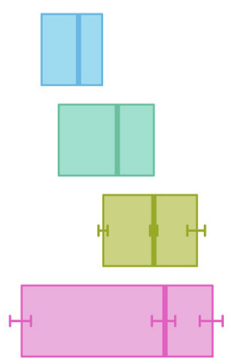

$1 \overline{\mathrm{ms}}$

Figure 2. Mormyrids generate species-specific EODs. A, Cladogram based on consensus trees of the species studied (Sullivan et al., 2000; Lavoué et al., 2003; Feulner et al., 2008; Sukhum et al., 2018). EOD traces are plotted as overlays of waveforms recorded from $N$ individuals of each species and aligned to peak 1 , defined as the head-positive peak. The EODs in C. numenius are displayed in three categories with distinct EOD durations (long, intermediate, and short). Right dotted box represents expanded EODs for all other species. $\boldsymbol{B}$, Box plots of EOD durations from each species, sorted by EOD duration. C, Power spectra of EODs from each species, from the same individuals shown in $A$. Each trace represents the average EOD power spectrum from 1 individual. $D$, Summary of EOD power spectra. Each bold line inside the box indicates the mean peak power frequency. The box limits indicate the mean lower and higher frequencies $3 \mathrm{~dB}$ below the peak. Error bars indicate SEM.

evoked potentials in the long-EOD C. numenius were blocked when stimuli were delivered with a 5-6 ms delay following the EOD command (Fig. 3C).

Corollary discharge timing is correlated with EOD waveform We next asked whether species diversity in EOD waveform (Fig. 2) was correlated with species diversity in corollary discharge timing (Fig. 3). First, we tested the relationship between EOD duration and CDI duration (Fig. $4 A, B$ ). Inhibition duration in
ELa was positively correlated with EOD duration (Fig. $4 A$; estimated slope $=0.20 \mathrm{~ms} / \mathrm{ms}, 95 \% \mathrm{CI}=0.10-0.29 \mathrm{~ms} / \mathrm{ms}, t_{(5)}=5.1$, $p=0.004$; estimated intercept $=2.4 \mathrm{~ms}, 95 \% \mathrm{CI}=1.7-3.1 \mathrm{~ms}$, $\left.t_{(5)}=8.6, p=0.0004\right)$, whereas inhibition duration in ELp was not correlated with EOD duration (Fig. $4 B$; estimated slope $=0.01 \mathrm{~ms} /$ $\mathrm{ms}, 95 \% \mathrm{CI}=-0.29$ to $0.30 \mathrm{~ms} / \mathrm{ms}, t_{(5)}=0.05, p=0.96$; estimated intercept $=3.3 \mathrm{~ms}, 95 \% \mathrm{CI}=1.1-5.5 \mathrm{~ms}, t_{(5)}=3.9, p=0.01$ ).

The different timing of CDI in species with different EOD durations (Fig. $3 C, D$ ) suggested that corollary discharge onset 

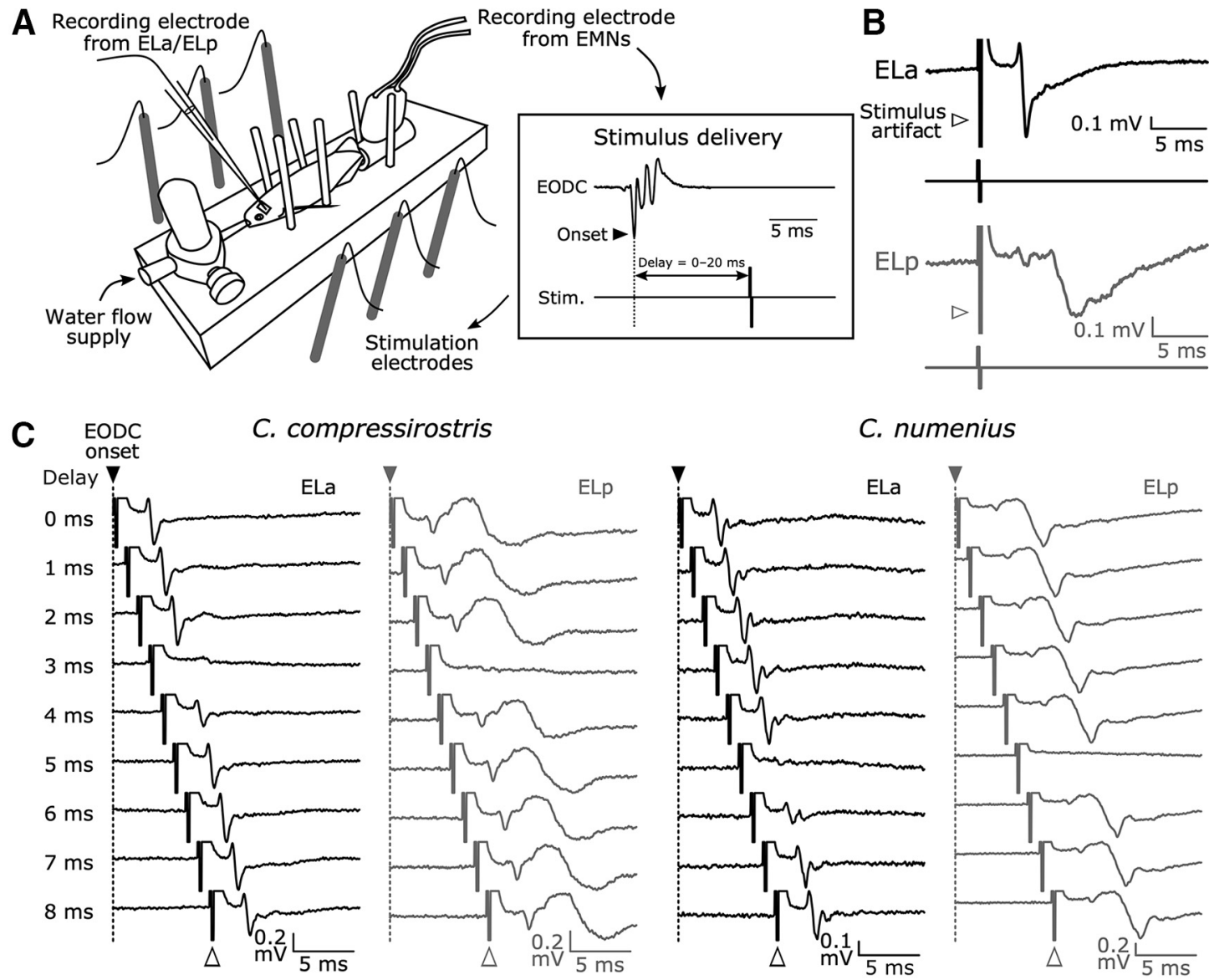

\section{C. numenius}

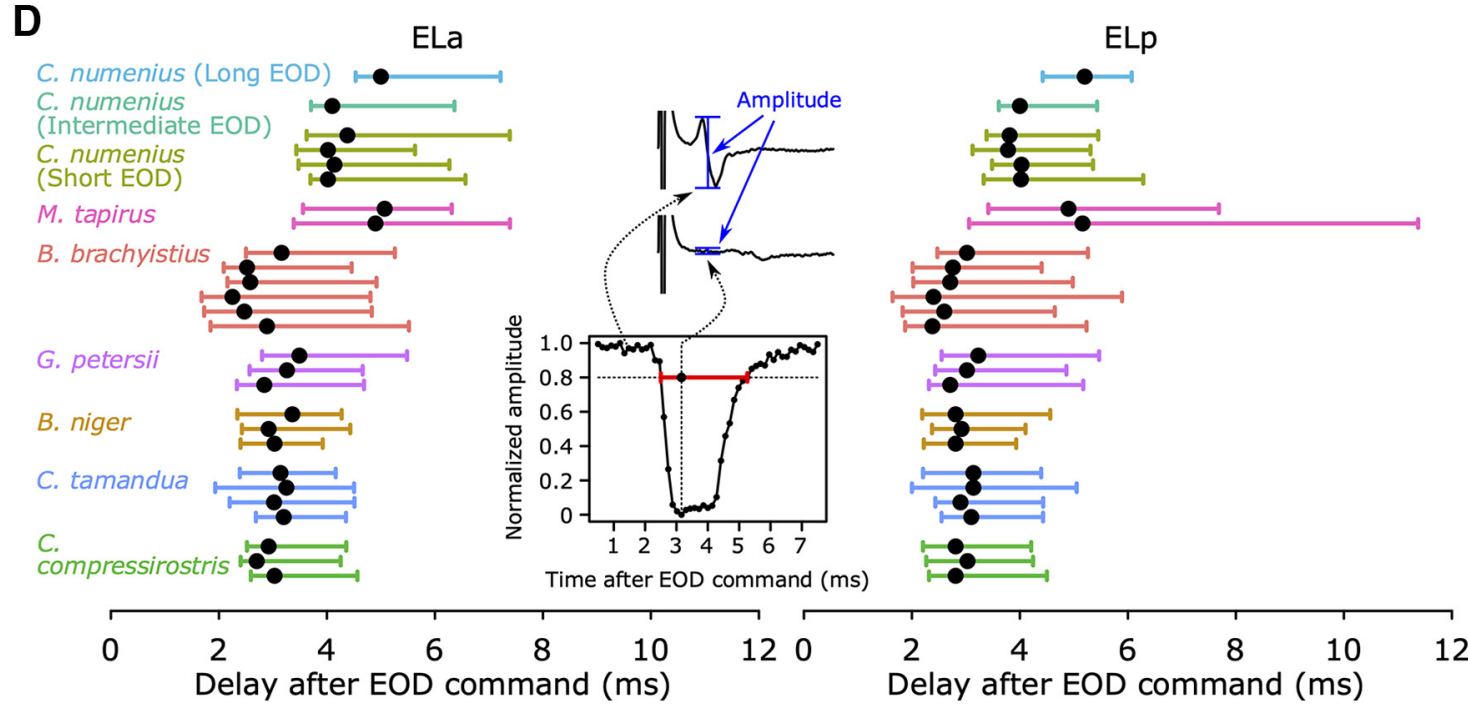

Figure 3. CDI in the communication circuit varies in timing and duration among mormyrid species. $\boldsymbol{A}$, Schematic representation of electrophysiological recording from the fish. An extracellular electrode inside a tube placed over the tail records EOD commands from spinal EMNs. To assess CDI related to EOD production, we delivered sensory stimuli at different delays ( 0 - 20 ms) after the EOD command (EODC) onset, which is determined as the first negative peak (indicated by black arrowhead), while recording evoked potentials in ELa or ELp. $\boldsymbol{B}$, Representative mean evoked potentials ( $n=10$ traces) obtained from ELa and ELp in M. tapirus. Stimulus artifact is indicated by open arrowheads. $C$, Representative mean evoked potentials in response to stimuli at varying delays following the EOD command (0-8 ms) in C. compressirostris and C. numenius (long EOD type). D, Summary of CDI across species. Inset, Measurement of CDI timing. The response magnitudes of evoked potentials were calculated as the peak-to-peak amplitude (blue bars) and normalized to the maximum and minimum peak-to-peak amplitudes across all stimulus delays. Using an $80 \%$ threshold, we determined inhibition onset, offset, and duration (red bar). The large point on the red bar indicates the peak inhibition time. Left and right panels represent the inhibition periods relative to the EOD command in ELa and ELp, respectively, across all individuals studied.

rather than duration might be associated with EOD duration. Indeed, we found that inhibition onset was strongly correlated with EOD duration in both ELa (Fig. 4C; estimated slope = $0.27 \mathrm{~ms} / \mathrm{ms}, 95 \% \mathrm{CI}=0.19-0.36 \mathrm{~ms} / \mathrm{ms}, t_{(5)}=8.0, p=0.0005$; estimated intercept $=2.4 \mathrm{~ms}, 95 \% \mathrm{CI}=1.8-3.1 \mathrm{~ms}, t_{(5)}=9.5 ; p=$ 0.0002 ) and ELp (Fig. 4D; estimated slope $=0.28 \mathrm{~ms} / \mathrm{ms}$, $95 \% \mathrm{CI}=0.21-0.35 \mathrm{~ms} / \mathrm{ms}, t_{(5)}=10.1, p=0.0002$; estimated intercept $\left.=2.3 \mathrm{~ms}, 95 \% \mathrm{CI}=1.8-2.8 \mathrm{~ms}, t_{(5)}=11.1, p=0.0001\right)$. 

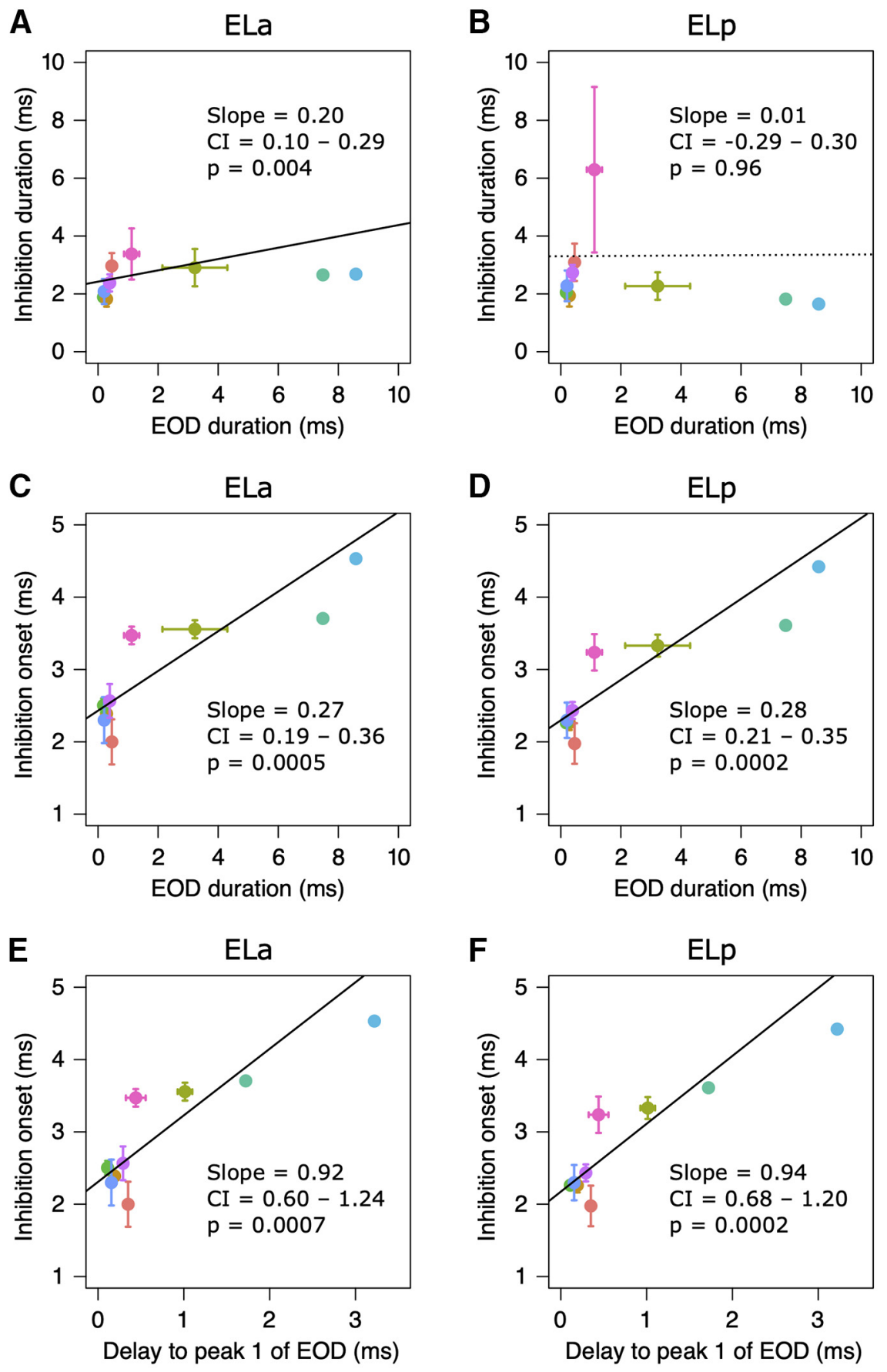
C. numenius (Intermediate EOD) G. petersii M. tapirus

Figure 4. $\mathrm{CDI}$ onset is correlated with EOD duration. $\boldsymbol{A}, \boldsymbol{B}$, Plots of inhibition duration ( $y$ axis) against EOD duration ( $x$ axis) in ELa and ELp. C, D, Plots of inhibition onset against EOD duration in ELa and ELp. $\boldsymbol{E}, \boldsymbol{F}$, Plots of inhibition onset against delay to peak 1 of the EOD in ELa and ELp. Points indicate the mean value of species \pm the SD (but not for $C$. numenius, which are divided into three groups based on EOD duration). Regression lines were determined using a PGLS analysis. Solid line indicates significant correlations. Dotted line indicates insignificant correlations. Although C. numenius is represented as three groups (long, intermediate, and short EOD), the regression was calculated using average values of each species. The estimated slope, $95 \% \mathrm{Cl}$, and the $p$ value are shown in each plot.

Here we recall two important features of $\mathrm{KO}$ responses to self-generated EODs: (1) each receptor responds with timelocked spikes to outside-positive changes in voltage across the skin; and (2) all KOs receive the same EOD waveform, which is inverted in polarity compared with the head-positive EOD recordings shown in Figure 2. This suggests that KOs should respond immediately after peak 1 of the EOD, when the self-generated EOD transitions from a negative to a positive peak. This further suggests that, to effectively block responses to self-generated EODs, the timing of CDI should also relate to the timing of EOD peak 1 . Indeed, we found that inhibition onset was strongly correlated with the delay to EOD peak 1 in both ELa (Fig. $4 E$; estimated slope $=0.92 \mathrm{~ms} / \mathrm{ms}, 95 \% \mathrm{CI}=$ $0.60-1.24 \mathrm{~ms} / \mathrm{ms}, t_{(5)}=7.5, p=0.0007$; estimated intercept $=2.3 \mathrm{~ms} ; 95 \%$ $\left.\mathrm{CI}=1.6-3.0 \mathrm{~ms}, t_{(5)}=8.4, p=0.0004\right)$ and ELp (Fig. 4F; estimated slope $=0.94 \mathrm{~ms} /$ $\mathrm{ms}, 95 \% \mathrm{CI}=0.68-1.20 \mathrm{~ms} / \mathrm{ms}, t_{(5)}=9.4$, $p=0.0002$; estimated intercept $=2.2 \mathrm{~ms}$, $95 \%$ CI $=1.6-2.7 \mathrm{~ms}, \quad t_{(5)}=9.8$, $p=0.0002$ ). The fact that the slopes were close to 1 indicates a 1:1 correspondence between the corollary discharge delay and delay to EOD peak 1 .

Since our definition of EOD duration requires arbitrary cutoffs to define the beginning and end of the EOD, we also determined whether EOD peak power frequency correlated with inhibition duration and onset. We found a significant negative correlation between peak power frequency and inhibition duration in ELa (estimated slope $=-0.14$ $\mathrm{ms} / \mathrm{kHz}, 95 \% \mathrm{CI}=-0.19$ to $-0.09 \mathrm{~ms} /$ $\mathrm{kHz}, t_{(5)}=-7.1, p=0.0008$; estimated intercept $=3.1 \mathrm{~ms}, 95 \% \mathrm{CI}=2.6-3.7 \mathrm{~ms}$, $t_{(5)}=13.9, p=3.4 \times 10^{-5}$ ), but this relationship was not significant in ELp (estimated slope $=-0.04 \mathrm{~ms} / \mathrm{kHz}, 95 \% \mathrm{CI}=$ -0.23 to $0.16 \mathrm{~ms} / \mathrm{kHz}, t_{(5)}=-0.47$, $p=0.66$; estimated intercept $=3.5 \mathrm{~ms}$, $\left.95 \% \mathrm{CI}=1.2-5.7 \mathrm{~ms}, t_{(5)}=3.9, p=0.01\right)$. By contrast, there were significant negative correlations between peak power frequency and inhibition onset in both ELa (estimated slope $=-0.19 \mathrm{~ms} / \mathrm{kHz}$, $95 \% \mathrm{CI}=-0.23$ to $-0.15 \mathrm{~ms} / \mathrm{kHz}, t_{(5)}=$ $-11.6, p=0.0001$; estimated intercept $=$ $3.4 \mathrm{~ms}, 95 \% \mathrm{CI}=2.9-3.9 \mathrm{~ms}, t_{(5)}=17.9$, $p=1.0 \times 10^{-5}$ ) and ELp (estimated slope $=-0.19 \mathrm{~ms} / \mathrm{kHz}, 95 \% \mathrm{CI}=-0.23$ to $-0.16 \mathrm{~ms} / \mathrm{kHz}, t_{(5)}=-14.8, p=2.5 \times$ $10^{-5}$; estimated intercept $=3.3 \mathrm{~ms}, 95 \%$ $\mathrm{CI}=2.9-3.7 \mathrm{~ms}, t_{(5)}=21.8, p=3.8 \times$ $\left.10^{-6}\right)$.

\section{KO responses to self-generated EODs} depend on the delay to peak 1

We tested the hypothesis that KO responses are time-locked to peak 1 of the EOD in both short-EOD and long-EOD species. We used previously published data from 6 species (Trzcinski, 2008; Trzcinski and Hopkins, 2008; Lyons-Warren et al., 2012; Baker 
et al., 2015) and examined the timing of $\mathrm{KO}$ spiking responses to conspecific EODs. By convention, "normal" EOD polarity is defined as a waveform recorded with the recording electrode at the head and a reference electrode at the tail as shown in Figure 2. Here we focused on KO responses to "inverted" EODs that represent the same waveform that KOs receive in response to self-generated EODs. We found that KOs responded with time-locked spikes with short delay following peak 1 of the EOD (Fig. $5 A$; delays between peak 1 of EOD and peak $\mathrm{KO}$ response were $0.14 \mathrm{~ms}$ in C. compressirostris, $0.07 \mathrm{~ms}$ in $B$. brachyistius, and $0.37 \mathrm{~ms}$ in C. numenius). Across species, delay to peak $\mathrm{KO}$ response strongly correlated with delay to peak 1 of the EOD (Fig. 5B; estimated slope $=1.15 \mathrm{~ms} / \mathrm{ms}, \quad 95 \% \quad \mathrm{CI}=1.07$ $1.23 \mathrm{~ms} / \mathrm{ms}, t_{(4)}=40.0, p=2.3 \times 10^{-6}$; estimated intercept $=0.09 \mathrm{~ms}, 95 \% \mathrm{CI}=-0.10$ to $0.28 \mathrm{~ms}$, $\left.t_{(4)}=1.3, p=0.26\right)$.

\section{CDI timing blocks KO responses to self-generated EODs}

Our results so far revealed that CDI and KO spiking responses were both correlated with delay to peak 1 of the EOD. This leads to the further question of whether the time-shifted CDI actually blocks responses to self-generated EODs. To address this question, we measured the delay between the EOD command from spinal electromotor neurons and the EOD in fish that were not electrically silenced and paralyzed. We found that delays between EOD command onset and EOD onset were similar among the 3 species $(C$. compressirostris, $3.12 \mathrm{~ms}$; B. brachyistius, $3.08 \mathrm{~ms}$; C. numenius, $3.28 \mathrm{~ms}$ ) (Fig. 6). Thus, the delays between EOD command onset and peak 1 of the EOD varied among the species (C. compressirostris, $3.24 \mathrm{~ms}$; B. brachyistius, $3.41 \mathrm{~ms}$; C. numenius, $5.05 \mathrm{~ms}$ ) (Fig. 6). Comparing the time courses of the EOD and CDI using an identical reference of the EOD command onset revealed that CDI overlapped with the timing of EOD peak 1 across species (Fig. 6).

\section{CDI timing is correlated with individual EOD waveform variation within $C$. numenius}

The high degree of individual variation in EOD duration in $C$. numenius (Fig. 2A,B) facilitates an examination of the correlation between EOD waveform and corollary discharge within species. Similar to our results across species, corollary discharge onset was strongly correlated with delay to peak 1 of the EOD in both ELa (Fig. 7; estimated slope $=0.43 \mathrm{~ms} / \mathrm{ms}$, 95\% CI $=0.24$ $0.62 \mathrm{~ms} / \mathrm{ms}, t_{(4)}=6.4, p=0.003$; estimated intercept $=3.1 \mathrm{~ms}$, $95 \% \mathrm{CI}=2.8-3.4 \mathrm{~ms}, t_{(4)}=27.0, p=1.1 \times 10^{-5}$ ) and ELp (Fig. 7; estimated slope $=0.48 \mathrm{~ms} / \mathrm{ms}, 95 \% \mathrm{CI}=0.25-0.70 \mathrm{~ms} / \mathrm{ms}, t_{(4)}=$ $5.8, p=0.004$; estimated intercept $=2.8 \mathrm{~ms}, 95 \%$ CI $2.4-3.2 \mathrm{~ms}$, $t_{(4)}=20.2, p=3.5 \times 10^{-5}$ ).

\section{Discussion}

Our findings provide evidence that diverse communication signals in mormyrids are correlated with CDI of the electrosensory pathway. We show that EOD duration is only weakly correlated with the duration of CDI, but strongly correlated with the onset of CDI (Fig. 4). Taking into account that electroreceptors (KOs)
B

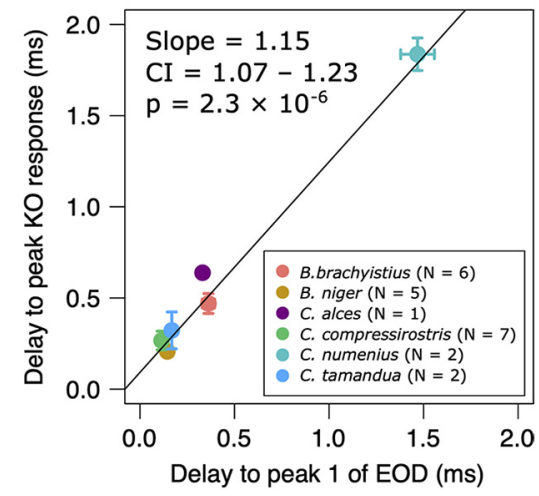

produce spikes with short latency following peak 1 of the EOD (Fig. 5) and that the CDI overlaps this peak (Fig. 6), we conclude that CDI has evolved to shift its time window so as to optimally block KO spikes in response to self-generated EODs (Fig. 8).

Evolutionary change in behavior can result from changes to sensory systems, motor systems, or both (Katz, 2011, 2016; Martin, 2012; Stöckl and Kelber, 2019). Correlated evolution of sensory and motor systems is especially apparent in communication systems, as this requires evolutionary change in both signal production and reception (Bass and Hopkins, 1984; Bass, 1986; Otte, 1992; ter Hofstede et al., 2015; Silva and Antunes, 2017). Indeed, mormyrids show correlated evolution between senders and receivers of their electric communication signals: the frequency tuning of KOs is related to the frequency spectrum of conspecific EODs (Bass and Hopkins, 1984; Lyons-Warren et al., 2012; but see also Baker et al., 2015). Here we add a further insight that signal evolution accompanies evolutionary change of neural circuitry underlying sensorimotor integration. Corollary discharges that filter sensory responses to self-generated signals are ubiquitous across communicating animals (Crapse and Sommer, 2008). In addition, signals among related species often vary widely in temporal features (Otte, 1992; Hopkins, 1999; Podos, 2001), and changing the timing of communication signals alters the timing of reafferent input. Therefore, we expect similar evolutionary change in corollary discharge timing to be widespread across sensory modalities and taxa. For example, the temporal structures of species-specific songs of crickets are similarly diverse to mormyrid EODs (Otte, 1992; ter Hofstede et al., 2015), and a similar CDI of the auditory pathway has been described in one species (Poulet and Hedwig, 2006).

Why does evolutionary change of EOD duration relate to the delay of CDI rather than inhibition duration? Theoretically, it is possible to alter the duration to cover the entirety of EODs with different durations. However, changing the delay of CDI without expanding the duration would avoid unnecessarily elongating the resulting insensitive period. This is because the KOs responding to self-generated EODs produce spikes only over a narrow window of time just after EOD peak 1 regardless of EOD duration (Fig. 5). Accordingly, we suggest an optimal evolutionary strategy for modifying CDI during signal evolution: minimizing the inhibitory window to only what is necessary for blocking receptor responses. 


\section{C. compressirostris}

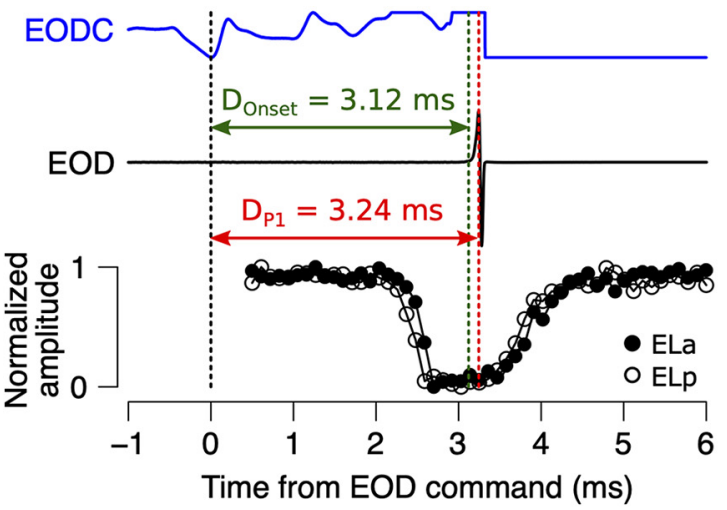

\section{B. brachyistius}

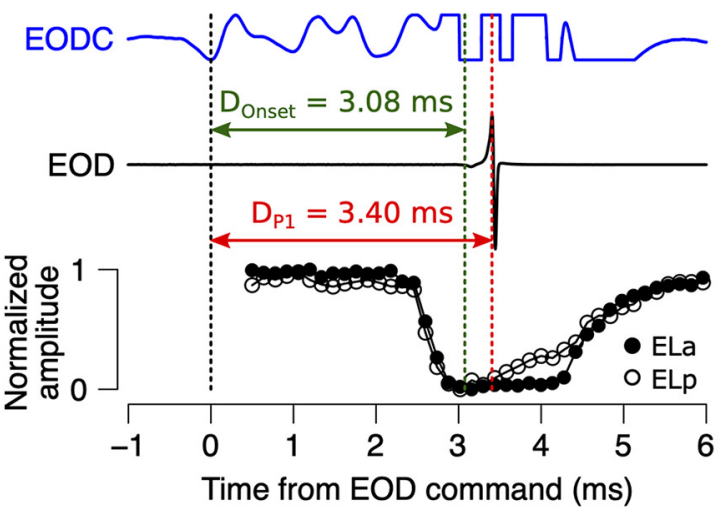

\section{C. numenius}

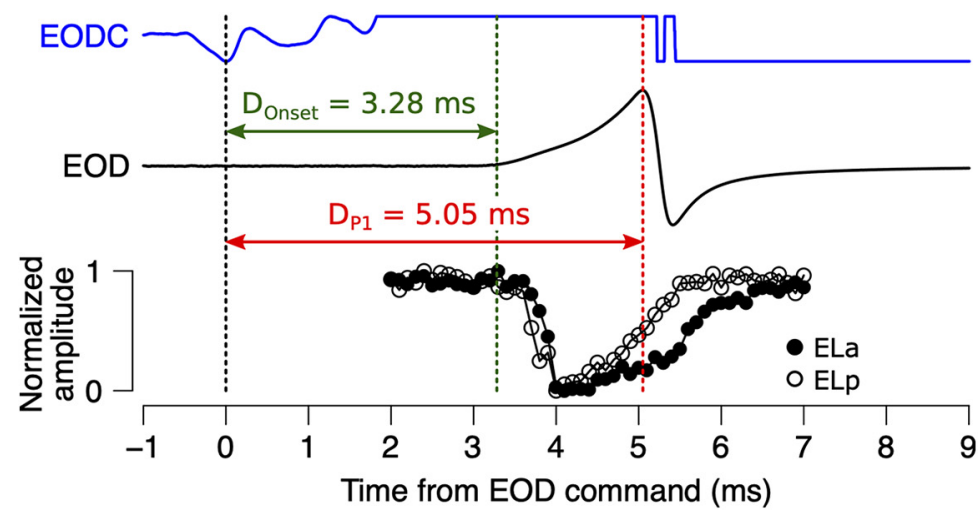

Figure 6. $C D I$ is timed to block responses to peak 1 of the EOD. We compared the time courses of the EOD command (EODC) (top blue traces), the EOD (middle traces), and CDI. Vertical dotted lines indicate EODC onset (black), EOD onset (green), and peak 1 of the EOD (red). $D_{\text {onset, }}$ Delay to EOD onset; $D_{P 1}$, delay to peak 1 of EOD.

Mormyrids have additional electrosensory systems, ampullary and mormyromast, used for passive and active electrolocation, respectively. Corollary discharge plays a significant, but different, role in these systems (von der Emde and Bell, 2003; Warren and Sawtell, 2016). The primary afferents of ampullary receptors are spontaneously active and exhibit long-lasting spiking responses that generally peak $>20 \mathrm{~ms}$ after the EOD (Bell and Russell, 1978). The primary afferents of mormyromasts have less spontaneous activity but exhibit long-lasting responses consisting of 2-8 spikes $\sim 2 \mathrm{~ms}$ after the EOD (Bell, 1990b). Corollary discharge in both systems works to subtract predictive sensory consequences of reafference by activating a modifiable efference copy (i.e., "negative image") through cerebellar-like circuitry in the ELL cortex (Warren and Sawtell, 2016). In this circuit, the negative image is generated in synapses between granule cells forming parallel fibers and principal cells through spike-timing-dependent plasticity (Bell et al., 1997). While the granule cells receive stereotyped corollary discharge inputs with short delays after the EOD command, their outputs are more temporally diverse and delayed (Kennedy et al., 2014). This is an important feature to provide a temporal basis for generating a sufficiently long negative image $(\sim 200 \mathrm{~ms})$. Species with longer EOD durations likely have longer-lasting responses, which would require even more temporal dispersion among granule cells to generate a longerlasting negative image. In addition, through a separate pathway, corollary discharge input facilitates responses to afferent input from mormyromasts, thereby selectively enhancing responses to reafferent EODs (Bell, 1990a). Here, too, species and individual differences in EOD duration may require corollary discharge input with different time courses.

What might be the source of species differences in the delay of CDI of the KO pathway? The command nucleus $(\mathrm{CN})$ drives the $\mathrm{EO}$ to produce each $\mathrm{EOD}$ through the medullary relay nucleus $(\mathrm{MRN})$ and the EMN (Fig. 1A). The CN also provides CDI to the nELL through the bulbar command-associated nucleus (BCA), the mesencephalic commandassociated nucleus (MCA), and the sublemniscal nucleus (slem) (Fig. 1A). The EOD command waveform recorded from the EMN is almost identical across species and is independent of EOD duration (Fig. 6) (Bennett, 1971; Bass and Hopkins, 1983; Grant et al., 1986; Carlson, 2002b), suggesting that command circuitry does not contribute to corollary discharge delays. Thus, the corollary discharge pathway must be adjusting the corollary discharge delay (Fig. 1A) (Bell et al., 1983). Since the BCA is involved in EOD command circuitry (Fig. 1A), it is an unlikely locus for evolutionary change. In addition, if the BCA contributes to the regulation of corollary discharge delay, this should influence all corollary discharge pathways (Bell et al., 1983). In contrast to KO, ampullary afferents respond to both outside-negative and outside-positive changes in voltage across the skin, and exhibit much longer-lasting responses (Bell and Russell, 1978). This indicates that the ampullary pathway requires different corollary discharge timing from the KO pathway. The MCA is an interesting candidate because it projects to precommand pathways that are involved in controlling the interpulse interval between EODs, and longer EODs require longer interpulse intervals (von der Emde et al., 
2000; Carlson, 2002a,b, 2003). In addition, the MCA indirectly provides corollary discharge excitatory input to the cells that receive input from mormyromast primary afferents, and this requires similarly precise timing as in the $\mathrm{KO}$ pathway (Zipser and Bennett, 1976; Bell and von der Emde, 1995; Bell et al., 1995). Furthermore, corollary discharge-related processing in the ampullary pathway is handled by a separate pathway that does not involve MCA (Bell et al., 1983). Thus, a single delay mechanism operating in MCA could coordinate appropriate delays to the KO pathway, mormyomast pathway, and precommand pathway, without affecting the ampullary pathway.

It is also possible that a change in slem,

which receives excitatory input from MCA and directly inhibits nELL, could contribute to regulating the corollary discharge delay we observed here. Future studies will compare this corollary discharge pathway across species to identify the source of species differences in delay.

What might be the mechanism for species differences in the CDI delay? There are at least three types of modifications that could change this delay. First, elongating axons could increase transmission delays much like the "delay lines" observed in several sensory systems (Carr and Konishi, 1988; Lyons-Warren et al., 2013b). Second, decreasing myelination and/or smaller axonal diameter could reduce the conduction velocity of axonal action potential propagation (Waxman et al., 1972; Seidl, 2014). Third, intrinsic properties of neurons (e.g., A-current, kinetics of transient outward $\mathrm{K}^{+}$current) can also affect inhibition timing (Getting, 1983). A combination of physiological recording and anatomic characterization of the corollary discharge pathway across species will uncover the mechanistic basis for evolutionary change in CDI delays.

In addition to species differences, we show that individual differences in corollary discharge delay are correlated with delay to EOD peak 1 in C. numenius (Fig. 6). A previous study demonstrated that EOD duration changes substantially with growth in C. numenius, and this correlates with ontogenetic changes in EO anatomy (Paul et al., 2015). For many mormyrid species, EOD waveform varies with ontogeny, sex, relative dominance, and season (Bass, 1986; Hopkins, 1999; Carlson et al., 2000; Werneyer and Kramer, 2006). The existence of individual variation in corollary discharge delays raises the question whether the same mechanism is used for individual variation of corollary discharge as for species differences. Moreover, are changes in corollary discharge timing and EOD duration mediated by a shared central regulatory pathway, or through neuronal plasticity associated with changing sensory input in response to self-generated EODs?

There are at least three possibilities that might explain how corollary discharge delay changes along with individual or evolutionary change in EOD waveform: (1) EOD command pathway drives both changes in EOD waveform and corollary discharge delay; (2) genetic regulation drives both changes; and (3) neural plasticity adapts the corollary discharge to changes in EOD duration. (1) would be impossible because there is no way for the electromotor network to provide the corollary discharge pathway with waveform information. EOD waveform is determined by the biophysical characteristics of electrocytes (Bennett, 1971;
ELa

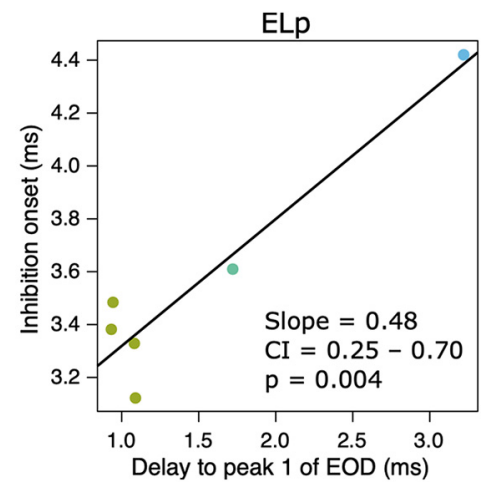
Slope $=0.43$ CI $=0.24-0.62$ $\mathrm{p}=0.003$

Figure 7. Corollary discharge onset is correlated with individual EOD waveform variation among C. numenius. Plots of inhiition onset ( $y$ axis) against delay to peak 1 of EOD ( $x$ axis) in ELa and ELp. Points indicate individual values. Regression lines

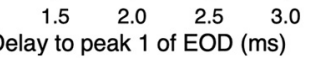

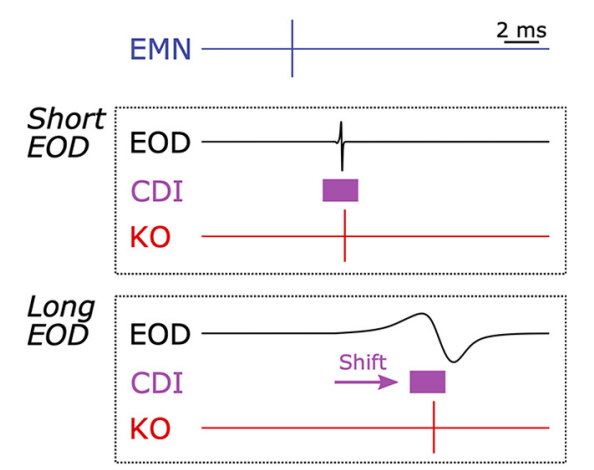

Figure 8. Time shift of CDI underlies communication signal evolution in mormyrids. Summary of corollary discharges between mormyrids with short-duration EODs and long-duration EODs. The schematic diagram represents spike timings of EMN and KO as well as EODs. The purple rectangles represent time windows of CDI.

Bass, 1986), independent from the EOD command (Bass and Hopkins, 1983). (2) would be possible. Recently, the genomic basis of EO anatomy and physiology is being increasingly well studied (Gallant et al., 2014; Gallant and O'Connell, 2020). It would be interesting if a central regulatory mechanism led to correlated transcriptional changes in the EO and corollary discharge pathway. (3) would be possible. Although previous studies suggest that corollary discharge in the KO system is invariant over several hours of electrophysiological experimentation, this was under a limited, unnatural situation in which the EOD was absent and no association with an alternative EOD was tested (Bell and Grant, 1989). Such an associative mechanism possibly takes place in the nELL as it is only site at which corollary discharge and $\mathrm{KO}$ sensory processing converge (Mugnaini and Maler, 1987). However, it is possible that retrograde signals could drive changes at earlier stages in the corollary discharge pathway.

The interspecies variance of corollary discharge delays had a 1:1 correspondence with delays to EOD peak 1, because the slopes of the regression lines were nearly 1 (Fig. $4 E, F$ ). By contrast, for intraspecies variance in $C$. numenius, these slopes were $<0.5$ (Fig. 7), indicating that fish with longer EODs have earlier corollary discharge delays than would be expected from the delay to EOD peak 1 . The reason remains unclear, but one possibility is that a plasticity-based mechanism governs a shift in the corollary discharge delay, and there may be a "lag time" in the response of the corollary discharge circuit to EOD elongation during development. 
Here we show evolutionary change of CDI for the first time. Our results strongly suggest a corollary discharge pathway makes an appropriate delay to block receptor responses to self-generated signals. Future studies will seek to identify the source of delays and the cellular mechanisms using electrophysiological and anatomic approaches. Furthermore, it will be interesting to study time shifts of CDI during signal development within individuals. Such studies will reveal the mechanisms by which sensorimotor integration is adjusted to account for species and individual differences in behavior.

\section{References}

Amagai S (1998) Time coding in the midbrain of mormyrid electric fish: II. Stimulus selectivity in the nucleus exterolateralis pars posterior. J Comp Physiol A 182:131-143.

Arnegard ME, McIntyre PB, Harmon LJ, Zelditch ML, Crampton WG, Davis JK, Sullivan JP, Lavoué S, Hopkins CD (2010) Sexual signal evolution outpaces ecological divergence during electric fish species radiation. Am Nat 176:335-356.

Baker CA, Kohashi T, Lyons-Warren AM, Ma X, Carlson BA (2013) Multiplexed temporal coding of electric communication signals in mormyrid fishes. J Exp Biol 216:2365-2379.

Baker CA, Huck KR, Carlson BA (2015) Peripheral sensory coding through oscillatory synchrony in weakly electric fish. eLife 4:e08163.

Bass AH (1986) Species differences in electric organs of mormyrids: substrates for species-typical electric organ discharge waveforms. J Comp Neurol 244:313-330.

Bass AH, Hopkins CD (1983) Hormonal control of sexual differentiation: change of electric organ discharge waveform. Science 220:971-974.

Bass AH, Hopkins CD (1984) Shifts in frequency tuning of electroreceptors in androgen-treated mormyrid fish. J Comp Physiol A 155:713-724.

Bell CC (1989) Sensory coding and corollary discharge effects in mormyrid electric fish. J Exp Biol 146:229-253.

Bell CC (1990a) Mormyromast electroreceptor organs and their afferent fibers in mormyrid fish: II. Intra-axonal recordings show initial stages of central processing. J Neurophysiol 63:303-318.

Bell CC (1990b) Mormyromast electroreceptor organs and their afferent fibers in mormyrid fish: III. Physiological differences between two morphological types of fibers. J Neurophysiol 63:319-332.

Bell CC, Grant K (1989) Corollary discharge inhibition and preservation of temporal information in a sensory nucleus of mormyrid electric fish. J Neurosci 9:1029-1044.

Bell CC, Russell CJ (1978) Effect of electric organ discharge on ampullary receptors in a mormyrid. Brain Res 145:85-96.

Bell C, von der Emde G (1995) Electric organ corollary discharge pathways in mormyrid fish: II. The medial juxtalobar nucleus. J Comp Physiol A 177:463-479.

Bell CC, Libouban S, Szabo T (1983) Pathways of the electric organ discharge command and its corollary discharges in mormyrid fish. J Comp Neurol 216:327-338.

Bell C, Dunn K, Hall C, Caputi A (1995) Electric organ corollary discharge pathways in mormyrid fish: I. The mesencephalic command associated nucleus. J Comp Physiol A 177:449-462.

Bell CC, Han VZ, Sugawara Y, Grant K (1997) Synaptic plasticity in a cerebellum-like structure depends on temporal order. Nature 387:278-281.

Bennett MVL (1965) Electroreceptors in mormyrids. Cold Spring Harb Symp Quant Biol 30:245-262.

Bennett MVL (1971) Electric organs. Fish Physiol 5:347-491.

Carlson BA (2002a) Electric signaling behavior and the mechanisms of electric organ discharge production in mormyrid fish. J Physiol (Paris) 96:405-419.

Carlson BA (2002b) Neuroanatomy of the mormyrid electromotor control system. J Comp Neurol 454:440-455.

Carlson BA (2003) Single-unit activity patterns in nuclei that control the electromotor command nucleus during spontaneous electric signal production in the mormyrid Brienomyrus brachyistius. J Neurosci 23:1012810136

Carlson BA (2009) Temporal-pattern recognition by single neurons in a sensory pathway devoted to social communication behavior. J Neurosci 29:9417-9428
Carlson BA, Hopkins CD, Thomas P (2000) Androgen correlates of socially induced changes in the electric organ discharge waveform of a mormyrid fish. Horm Behav 38:177-186.

Carlson BA, Hasan SM, Hollmann M, Miller DB, Harmon LJ, Arnegard ME (2011) Brain evolution triggers increased diversification of electric fishes. Science 332:583-586.

Carr CE, Konishi M (1988) Axonal delay lines for time measurement in the owl's brainstem. Proc Natl Acad Sci USA 85:8311-8315.

Crapse TB, Sommer MA (2008) Corollary discharge across the animal kingdom. Nat Rev Neurosci 9:587-600.

Ding Y, Lillvis JL, Cande J, Berman GJ, Arthur BJ, Long X, Xu M, Dickson BJ, Stern DL (2019) Neural evolution of context-dependent fly song. Curr Biol 29:1089-1099.

Einevoll GT, Kayser C, Logothetis NK, Panzeri S (2013) Modelling and analysis of local field potentials for studying the function of cortical circuits. Nat Rev Neurosci 14:770-785.

Feulner PG, Kirschbaum F, Tiedemann R (2008) Adaptive radiation in the Congo River: an ecological speciation scenario for African weakly electric fish (Teleostei; Mormyridae; Campylomormyrus). J Physiol (Paris) 102:340-346.

Gallant JR, O'Connell LA (2020) Studying convergent evolution to relate genotype to behavioral phenotype. J Exp Biol 223:jeb213447.

Gallant JR, Traeger LL, Volkening JD, Moffett H, Chen PH, Novina CD, Phillips GN, Anand R, Wells GB, Pinch M, Güth R, Unguez GA, Albert JS, Zakon HH, Samanta MP, Sussman MR (2014) Genomic basis for the convergent evolution of electric organs. Science 344:1522-1525.

Getting PA (1983) Mechanisms of pattern generation underlying swimming in Tritonia: III. Intrinsic and synaptic mechanisms for delayed excitation. J Neurophysiol 49:1036-1050.

Grafen A (1989) The phylogenetic regression. Philos Trans R Soc Lond B Biol Sci 326:119-157.

Grant K, Bell CC, Clausse S, Ravaille M (1986) Morphology and physiology of the brainstem nuclei controlling the electric organ discharge in mormyrid fish. J Comp Neurol 245:514-530.

Hopkins CD (1981) On the diversity of electric signals in a community of mormyrid electric fish in west Africa. Am Zool 21:211-222.

Hopkins CD (1999) Design features for electric communication. J Exp Biol 202:1217-1228.

Hopkins CD, Bass AH (1981) Temporal coding of species recognition signals in an electric fish. Science 212:85-87.

Jacob PF, Hedwig B (2019) Structure, activity and function of a singing CPG interneuron controlling cricket species-specific acoustic signaling. J Neurosci 39:96-111.

Katz PS (2011) Neural mechanisms underlying the evolvability of behaviour. Philos Trans R Soc Lond B Biol Sci 366:2086-2099.

Katz PS (2016) Evolution of central pattern generators and rhythmic behaviours. Philos Trans R Soc Lond B Biol Sci 371:20150057.

Kennedy A, Wayne G, Kaifosh P, Alviña K, Abbott L, Sawtell NB (2014) A temporal basis for predicting the sensory consequences of motor commands in an electric fish. Nat Neurosci 17:416-422.

Kwong-Brown U, Tobias ML, Elias DO, Hall IC, Elemans CP, Kelley DB (2019) The return to water in ancestral Xenopus was accompanied by a novel mechanism for producing and shaping vocal signals. eLife 8: e39946.

Lavoué S, Sullivan JP, Hopkins CD (2003) Phylogenetic utility of the first two introns of the S7 ribosomal protein gene in African electric fishes (Mormyroidea: teleostei) and congruence with other molecular markers. Biol J Linn Soc 78:273-292.

Lyons-Warren AM, Hollmann M, Carlson BA (2012) Sensory receptor diversity establishes a peripheral population code for stimulus duration at low intensities. J Exp Biol 215:2586-2600.

Lyons-Warren AM, Kohashi T, Mennerick S, Carlson BA (2013a) Retrograde fluorescent labeling allows for targeted extracellular singleunit recording from identified neurons in vivo. J Vis Exp 76:e3921.

Lyons-Warren AM, Kohashi T, Mennerick S, Carlson BA (2013b) Detection of submillisecond spike timing differences based on delay-line anticoincidence detection. J Neurophysiol 110:2295-2311.

Martin GR (2012) Through birds' eyes: insights into avian sensory ecology. J Ornithol 153:23-48.

Mugnaini E, Maler L (1987) Cytology and immunocytochemistry of the nucleus of the lateral line lobe in the electric fish Gnathonemus petersii 
(mormyridae): evidence suggesting that GABAergic synapses mediate an inhibitory corollary discharge. Synapse 1:32-56.

Mundry R (2014) Statistical issues and assumptions of phylogenetic generalized least squares. In: Modern phylogenetic comparative methods and their application in evolutionary biology, concepts and practice (Garamszegi LZ, ed), pp 131-153. Berlin: Springer.

Osorio D, Vorobyev M (2008) A review of the evolution of animal colour vision and visual communication signals. Vision Res 48:2042-2051.

Otte D (1992) Evolution of cricket songs. J Orthop Res 1:25-49.

Paradis E, Schliep K (2019) ape 5.0: an environment for modern phylogenetics and evolutionary analyses in R. Bioinformatics 35:526-528.

Paul C, Mamonekene V, Vater M, Feulner PG, Engelmann J, Tiedemann R, Kirschbaum F (2015) Comparative histology of the adult electric organ among four species of the genus Campylomormyrus (Teleostei: mormyridae). J Comp Physiol A 201:357-374

Pinheiro J, Bates D, DebRoy S, Sarkar D, R Core Team (2020) nlme: linear and nonlinear mixed effects models. R package version 3.1-145. https:// CRAN.R-project.org $/$ package $=$ nlme.

Podos J (2001) Correlated evolution of morphology and vocal signal structure in Darwin's finches. Nature 409:185-188.

Poulet JFA, Hedwig B (2006) The cellular basis of a corollary discharge. Science 311:518-522.

Poulet JFA, Hedwig B (2007) New insights into corollary discharges mediated by identified neural pathways. Trends Neurosci 30:14-21.

Russell CJ, Bell CC (1978) Neuronal responses to electrosensory input in mormyrid valvula cerebelli. J Neurophysiol 41:1495-1510.

Schneider DM, Mooney R (2018) How movement modulates hearing. Annu Rev Neurosci 41:553-572.

Seeholzer LF, Seppo M, Stern DL, Ruta V (2018) Evolution of a central neural circuit underlies Drosophila mate preferences. Nature 559:564-569.

Seidl AH (2014) Regulation of conduction time along axons. Neuroscience 276:126-134.

Silva L, Antunes A (2017) Vomeronasal receptors in vertebrates and the evolution of pheromone detection. Annu Rev Anim Biosci 5:353-370.

Sperry RW (1950) Neural basis of the spontaneous optokinetic response produced by visual inversion. J Comp Physiol Psychol 43:482-489.

Stöckl AL, Kelber A (2019) Fuelling on the wing: sensory ecology of hawkmoth foraging. J Comp Physiol A 205:399-413.

Straka H, Simmers J, Chagnaud BP (2018) A new perspective on predictive motor signaling. Curr Biol 28:R232-R243.

Sukhum KV, Shen J, Carlson BA (2018) Extreme enlargement of the cerebellum in a clade of teleost fishes that evolved a novel active sensory system. Curr Biol 28:3857-3863.
Sullivan JP, Lavoué S, Hopkins CD (2000) Molecular systematics of the African electric fishes (Mormyroidea: teleostei) and a model for the evolution of their electric organs. J Exp Biol 203:665-683.

ter Hofstede HM, Schöneich S, Robillard T, Hedwig B (2015) Evolution of a communication system by sensory exploitation of startle behavior. Curr Biol 25:3245-3252.

Trzcinski N (2008) Electric signaling and Knollenorgan receptor organ properties in the genus Campylomormyrus (Mormyridae). Neurobiology and Behavior Honors Thesis, Cornell University.

Trzcinski N, Hopkins CD (2008) Electric signaling and electroreception properties in electric fishes of the genus Campylomormyrus (Mormyridae). Cornell Synapse 2:19-22.

Vélez A, Carlson BA (2016) Detection of transient synchrony across oscillating receptors by the central electrosensory system of mormyrid fish. eLife 5:e16851.

Vélez A, Kohashi T, Lu A, Carlson BA (2017) The cellular and circuit basis for evolutionary change in sensory perception in mormyrid fishes. Sci Rep 7:3783.

von der Emde G (1999) Active electrolocation of objects in weakly electric fish. J Exp Biol 202:1205-1215.

von der Emde G, Bell CC (2003) Active electrolocation and its neural processing in mormyrid electric fishes. In: Sensory processing in aquatic environments (Collin SP, Marshall J, eds), pp 92-107. New York: Springer.

von der Emde G, Sena LG, Niso R, Grant K (2000) The midbrain precommand nucleus of the mormyrid electromotor network. J Neurosci 20:5482-5495.

von Holst E, Mittelstaedt H (1950) Das reafferenzprinzip. Naturwissenschaften 37:464-476.

Warren R, Sawtell NB (2016) A comparative approach to cerebellar function: insights from electrosensory systems. Curr Opin Neurobiol 41:31-37.

Waxman SG, Pappas GD, Bennett MVL (1972) Morphological correlates of functional differentiation of nodes of Ranvier along single along single fibers in the neurogenetic electric organ of the knife fish Sternarchus. J Cell Biol 53:210-224.

Werneyer M, Kramer B (2006) Ontogenetic development of electric-organ discharges in a mormyrid fish, the bulldog Marcusenius macrolepidotus (South African form). J Fish Biol 69:1190-1201.

Xu-Friedman M, Hopkins CD (1999) Central mechanisms of temporal analysis in the Knollenorgan pathway of mormyrid electric fish. J Exp Biol 202:1311-1318.

Zipser B, Bennett MVL (1976) Interaction of electrosensory and electromotor signals in lateral line lobe of a mormyrid fish. J Neurophysiol 39:713-721. 